\section{Drug Use Evaluation of Endocrine Therapy in Women with Breast Cancer in a Chinese Hospital}

Prescription drugs constitute an important component of health care. However, drugs can benefit patients only if they are used appropriately. Drug use evaluation (DUE) can detect inappropriate and/or unnecessarily costly drug therapy and thereby promote the rational use of drugs. ${ }^{1}$ Because DUE programs are rarely conducted in Chinese hospitals today, there is a paucity of information on the quality of prescribing, and there is no organization that advocates the conduct of DUEs in China.

Breast cancer is the most common cancer and the leading cause of cancer death in women worldwide, accounting for $23 \%$ of the total new cancer cases and $14 \%$ of the total cancer deaths in 2008. ${ }^{2}$ Evidence-based guidelines for breast cancer have been developed and disseminated both internationally and in China. Adjuvant endocrine therapy has significantly reduced breast cancer mortality. ${ }^{3,4,5}$ Thus, adherence to treatment guidelines has an important impact on public health.

In this study, we conducted a retrospective DUE to assess the quality of endocrine therapy prescribing in patients with breast cancer during the 3-year period from January 1, 2007, through December 31, 2009, in a Chinese hospital. The hospital is the largest maternal and child health hospital in Nanjing, with 632 total beds, 40 of which are in the breast cancer department.

All patients diagnosed with breast cancer were included in the study and evaluated only once. This study was approved by the ethics committee of the hospital, and data collection was confined to information recorded in the electronic patient record (EPR) and hospital information system (HIS). The DUE criteria were developed from the 2007-2009 National Comprehensive Cancer Network (NCCN) Clinical Practice Guidelines in Breast Cancer (Chinese Version). 3,4,5 The NCCN guidelines call for the determination of estrogen receptor (ER) and progesterone receptor (PR) content in all primary invasive breast cancer. Patients with invasive breast cancers that are ER- or PR-positive should be considered for adjuvant endocrine therapy, and aromatase inhibitors should not be used in the treatment of premenopausal women with breast cancer. ${ }^{3,4,5}$

There were 192 breast cancer patients treated in the 3-year period. All patients had invasive breast cancer and had ER and PR content determined. We found that an unacceptably high $20.3 \% \quad(n=39)$ of these patients received inappropriate drug therapy in this hospital; 27 patients (14.1\%) whose cancers were ER- or PR-positive did not receive endocrine therapy as suggested by NCCN guidelines, and 12 premenopausal patients $(6.3 \%)$ received aromatase inhibitors. Endocrine therapy should be prescribed to patients with ER- or PR-positive cancers, regardless of patient age, lymph node status, or whether adjuvant chemotherapy is to be administered. ${ }^{5}$ Aromatase inhibitors are not active in the treatment of women with functioning ovaries and should not be used in women whose ovarian function cannot be reliably assessed because of treatment-induced amenorrhea. ${ }^{5}$

We provided the DUE results to the director of the breast cancer department and suggested that physicians should be provided with additional education on life-saving procedures, such as adjuvant systemic treatments, in cancer medicine. In a department meeting, the director stressed these issues and requested that physicians correct these irrational drug use problems.

A follow-up reevaluation was done 6 months after the intervention in the same hospital. We collected data for all 37 breast cancer patients treated from July 1, 2010, to February 28,2011 . The results showed that 4 of 37 patients (10.8\%) were not treated according to guidelines. Of those, 2 patients (5.4\%) with ER- or PR-positive cancers did not receive endocrine therapy, and aromatase inhibitors were prescribed for 2 premenopausal patients (5.4\%). Although there was still inappropriate drug therapy, the DUE process and feedback were associated with smaller proportions of breast cancer patients with ER- or PR-positive tumors who did not receive endocrine therapy and with reduced prescribing of aromatase inhibitors in premenopausal patients.

This DUE study helped to identify and correct medicationrelated problems during adjuvant endocrine therapy in women with breast cancer, and we suggest that the national health authorities in China consider a requirement for the conduct of standardized DUE programs in oncology in Chinese hospitals.

\section{Yanni Chen, Phar M \\ Clinical Pharmacist \\ chenyanni96@sina.com}

\section{Li Wang, Phar B}

Director of Pharmacy Department

wl_wqf@126.com

\section{Cheng Lu, MD, PhD}

Director of Breast Cancer Department

lucheng66@126.com

Affiliated Hospital of Nanjing Medical University Nanjing Maternal and Child Health Hospital, Nanjing, China 210004.

\section{DISCLOSURES}

There was no external funding for this research. The authors report no financial or other potential conflicts of interest related to this letter.

\section{REFERENCES}

1. World Health Organization. Introduction to drug utilization research. 2003. Available at: http://apps.who.int/medicinedocs/en/d/Js4876e/2.2.html. Accessed June 27, 2011

2. Jemal A, Bray F, Center MM, Ferlay J, Ward E, Forman D. Global cancer statistics. CA Cancer J Clin. 2011;61(2):69-90. Available at: http://onlinelibrary.wiley.com/doi/10.3322/caac.20107/pdf. Accessed June 27, 2011.

3. NCCN Clinical Practice Guidelines in Breast Cancer (Chinese Version), 2007 (version 1.0). Available at: http://wenku.baidu.com/ view/5cc7fd995le79b89680226b4.html. Accessed June 27, 2011.

4. NCCN Clinical Practice Guidelines in Breast Cancer (Chinese Version), 2008 (version 1.0). Available at: http://www.docin.com/p-7417920.html. Accessed June 27, 2011

5. NCCN Clinical Practice Guidelines in Breast Cancer (Chinese Version) 2009 (version 1.0). Available at: http://www.docin.com/p-34244729.html. Accessed June 23, 2011 